\title{
Optimization of neutrino fluxes for European Super-Beams
}

\section{A. Longhin*}

Irfu, CEA-Saclay, 91191 Gif-sur-Yvette, France

E-mail: andrea. longhinecea.fr

We present an optimization of neutrino beams which could be produced at CERN and aimed to a set of seven underground sites in Europe with distances ranging from $130 \mathrm{~km}$ to $2300 \mathrm{~km}$. Realistic studies on the feasibility of a next generation very massive neutrino observatory exist for these sites in the context of the EU LAGUNA design study. We consider precise scenarios for the proton driver and the far detector. The flux calculation profits of a full GEANT4 simulation which has been recently developed. A powerful optimization procedure based on the achievable sensitivity on $\sin ^{2} 2 \theta_{13}$ has been employed. A comparison between the neutrino oscillation physics potential of each baseline based on a coherent set of tools is presented.

35th International Conference of High Energy Physics - ICHEP2010,

July 22-28, 2010

Paris France

* Speaker. 
The feasibility of a European next-generation very massive neutrino observatory in seven potential candidate sites located at distances from CERN ranging from $130 \mathrm{~km}$ to $2300 \mathrm{~km}$, has been studied within the LAGUNA FP7 design study. The considered underground sites are: Fréjus (France) at $130 \mathrm{~km}$, Canfranc (Spain) at $630 \mathrm{~km}$, Caso (Italy) at $665 \mathrm{~km}$, Sierozsowice (Poland) at $950 \mathrm{~km}$, Boulby (UK) at $1050 \mathrm{~km}$, Slanic (Romania) at $1570 \mathrm{~km}$ and Pyhäsalmi (Finland) at $2300 \mathrm{~km}$. When coupled to powerful and well-tuned neutrino beams from CERN, large detectors hosted in such sites, would measure with unprecedented sensitivity the last unknown mixing angle $\theta_{13}$, explore CP violating effects in the leptonic sector and determine the neutrino mass ordering (hierarchy). For this study the following scenarios for the accelerators and far detectors have been considered:

- for $L=130 \mathrm{~km}$ a super-conducting linac operating at $4.5 \mathrm{GeV}$ (HP-SPL [1]) providing 0.56 . $10^{23}$ protons on target (POT) per year, associated with a 440 kton Water Čerenkov detector (MEMPHYS [2]);

- for longer baselines a synchrotron at $50 \mathrm{GeV}$ (HP-PS2 [3]) providing $3 \cdot 10^{21}$ POT/year aimed towards a 100 kton Liquid Argon Time Projection Chamber (GLACIER [4]).

The optimization of the neutrino fluxes for the CERN-Fréjus baseline has been the subject of an extensive study within the EURO $v$ Design Study [5]. We will then use those results and describe in more detail the optimization for the longer baselines.

The simulation of the proton-target interactions and of the $v$ fluxes is based on the GEANT4 libraries in association with the QGSP hadronic package. The target is modeled as a $1 \mathrm{~m}$ long cylinder of graphite $\left(\rho=1.85 \mathrm{~g} / \mathrm{cm}^{3}\right)$ with $4 \mathrm{~mm}$ diameter. The secondary mesons focusing is based on a pair of sequential magnetic horns (horn and reflector hereafter) whose shapes in the radial plane are parametrized using four quadratic functions. Other additional parameters of the system are: the horn-reflector distance $\left(\Delta_{H R}\right)$, the geometry of the cylindrical decay tunnel $\left(L_{T}\right.$, $\left.r_{T}\right)$, the longitudinal position of the target $\left(z_{t}\right)$ and the currents circulating in the horn and the reflector $\left(i_{H}, i_{R}\right)$. The optimization procedure was performed separately for each site, the guiding line being the final sensitivity which could be obtained for $\sin ^{2} 2 \theta_{13}$. More precisely we introduced as a figure of merit of the focusing system, the quantity $\lambda$ defined as the $\delta_{C P}$-averaged $99 \%$ C.L. sensitivity limit on $\sin ^{2} 2 \theta_{13}$. The procedure consists in sampling the space of the focusing system parameters and performing the simulation of fluxes for each configuration to compute its figure of merit $\lambda$. By selecting a subset of configurations producing good exclusion limits (low values for $\lambda$ ), it is possible to pin-down the most effective parameters and, repeating the procedure on a limited region of parameters, converge towards better configurations.

In the first step, the horn shapes, the tunnel geometry and the currents were fixed $\left(L_{T}=300\right.$ $\mathrm{m}, r_{T}=1.5 \mathrm{~m}, i=200 \mathrm{kA}$ ) while $z_{t}$ and $\Delta_{H R}$ were varied independently in the ranges [-1.5, 2.5] $\mathrm{m}$ and $[0,300] \mathrm{m}$ respectively. We observed a marked dependence of $\lambda$ on $z_{t}$ while the dependence on $\Delta_{H R}$ is milder. After having chosen the best point in the $\left(\Delta_{H R}, z_{t}\right)$ space, the procedure was repeated for the the decay tunnel geometry $\left(L_{T}, r_{T}\right)$ in the ranges $[10,500] \mathrm{m}$ and $[0.3,3] \mathrm{m}$.

To cross check the previous procedure we also performed a more general scan of the configurations allowing all of other relevant parameters to vary independently at the same time. In particular the shape parameters of the horn and the reflector were sampled with uniform distribution within 
$50 \%$ of the central values which were taken as those describing the horns used for the NuMI beam. The importance of the $z_{t}$ variable was confirmed. This parameter is strongly correlated with the mean energy of the $v_{\mu}$ spectrum $\left(\left\langle E\left(v_{\mu}\right)\right\rangle\right)$ and the $\lambda$ parameter. With the used setup, moving the target upstream and downstream allows to explore values of $\left\langle E\left(v_{\mu}\right)\right\rangle$ between approximately 2 and $6 \mathrm{GeV}$. The correlation between $\lambda$ and $\left\langle E\left(v_{\mu}\right)\right\rangle$ is shown in the left hand plots of Fig. 1. It can be noticed that the optimal energies tend to roughly follow the position of the first maximum of the oscillation probability which for the considered baselines ranges from $0.26 \mathrm{MeV}$ up to $4.65 \mathrm{GeV}$ (vertical lines) for $\Delta m_{13}^{2}=2.5 \cdot 10^{-3} \mathrm{eV}^{2}$.
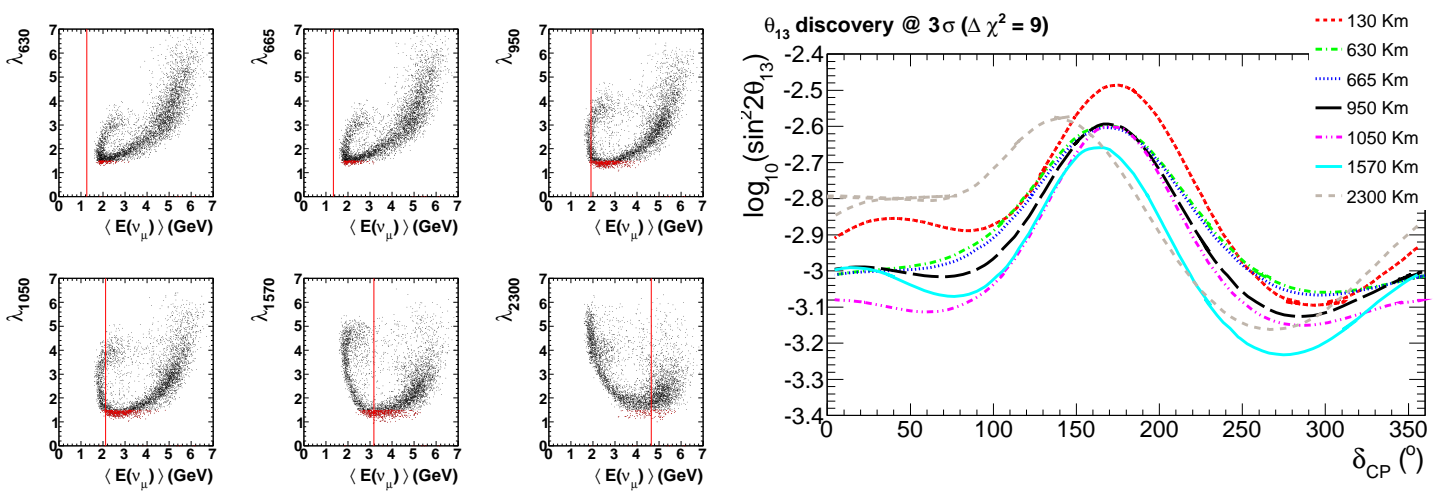

Figure 1: Left: correlation between $\lambda$ and $\left\langle E\left(v_{\mu}\right)\right\rangle$ for the six longer baselines. Each point corresponds to a configuration of the focusing system. Vertical lines mark the energy of the first oscillation maximum for each baseline. Right: discovery potential for $\theta_{13} \neq 0$ calculated with the GLoBES software. Running periods are 2(8) years in $v(\bar{v})$ mode. A systematic error on the fluxes of $5 \%$ has been assumed.

The discovery potential for $\sin ^{2} 2 \theta_{13} \neq 0$ for a set of tuned configurations lies in the $10^{-3}$ ballpark ( $3 \sigma$-level) as shown in the right hand plot of Fig. 1. More stringent limits are obtained with the 1050 and $1570 \mathrm{~km}$ baselines even though the difference with respect to the other choices is not very large. The discovery potential for CP violation (not shown) tends to reflect the performances in terms of $\theta_{13}$ exclusion especially in the region $\delta_{C P}<\pi$ which is ruled by the $\bar{v}$ data. The possibility to determine the mass hierarchy is instead strongly dependent on the use of very long baselines, as expected. The fluxes obtained with the optimized setups and further information are available in electronic format [6].

I would like to thank A. Meregaglia for providing the GLoBES AEDL description of the GLACIER detector.

\section{References}

[1] M. Baylac et al., CERN-2006-006.

[2] A. de Bellefon et al., arXiv:hep-ex/0607026.

[3] CERN PS2 working group. https://paf-ps2.web.cern.ch. A. Rubbia, arXiv:hep-ph/1003.1921v1.

[4] A. Rubbia, arXiv:hep-ph/0402110.

[5] A. Longhin, AIP Conf. Proc. 1222:339-343, 2010.

[6] A. Longhin, http://irfu.cea.fr/en/Phocea/Pisp/index.php?id=54. 